Copyright (C) 2012 IEEE. Personal use of this material is permitted. Permission from IEEE must be obtained for all other uses, in any current or future media, including reprinting/republishing this material for advertising or promotional purposes, creating new collective works, for resale or redistribution to servers or lists, or reuse of any copyrighted component of this work in other works. 


\section{Time-lapse borehole radar measurements in a sandy groundwater system during a winter recharge cycle}

\author{
E. Strobach*, B. D. Harris, J. C. Dupuis, A. W. Kepic \\ Dept. of Exploration Geophysics \\ Curtin University of Technology \\ Perth, Western Australia \\ *elmar.strobach@postgrad.curtin.edu.au
}

\author{
M. Martin \\ Water Corporation \\ Perth, Western Australia
}

\begin{abstract}
Borehole Radar has demonstrated to be an effective method to determine water content profiles within the vadose zone. Time-lapse measurements shown in this study were acquired on the Gnangara Mound north of Perth, Western Australia. Borehole radar experiments using vertical radar profiling and zero-offset crosswell profiling acquisition geometries have been performed under different soil moisture conditions. Time-lapse repeatability proved robust for zero-offset profiles, while vertical radar profiles provided mixed results. The variations observed in the zero-offset profiling are expected to be related to the seasonal variations of soil moisture content since results from the saturated zone remained constant during the tests. We quantify the wetting front developing through the dry soil profile on a monthly basis. Infiltration behaviours are characterized under different scenarios ranging from homogeneous soil moisture distribution with shallow water tables to heterogeneous soil profiles including water retentive layers and the watertable at $12 \mathrm{~m}$ depth. The measured soil moisture profiles and infiltration observations have implications for groundwater recharge estimation and unsaturated flow parameter estimation.
\end{abstract}

Keywords - Borehole radar; zero-offset profiling; vertical radar profiling, groundwater-recharge, unsatirated flow

\section{INTRODUCTION}

\section{A. Borehole Radar techniques}

The borehole radar (BHR) technique is widely used to quantify moisture content distribution of soil profiles (e.g. [1, $3,4]$ ). Transient unsaturated flow can be investigated using time-lapse measurements. From time-variant water content profiles, hydrologic trajectories can be inferred. Various crosswell acquisition geometries are available to the practitioner. Here, we concentrate on the Zero-Offset Profiling (ZOP) technique. This geometry provides one traveltime per depth interval as both transmitting (Tx) and receiving (Rx) antenna are lowered into adjacent boreholes at a constant speed and a zero vertical offset. The interpretation of ZOP firstarrivals can lead to false velocity estimates at large contrast interfaces where refracted energy occurs but direct travel is assumed ([9]). Reference [7] discusses pre-inversion corrections for borehole radar measurements which include time-variant zero-time correction, borehole deviation (spatial correction) and waveform distortion. We typically contribute waveform distortion to (a) frequency dependent attenuation in different dielectric media, (b) interfering wavefields or (c) null coupling ( $\mathrm{Tx} \perp \mathrm{Rx}$ ). Single-hole applications include zero- or multi-offset vertical radar profiling (VRP) ([13]) and commonoffset profiling. VRP interpretation, analogous to vertical seismic profiling, is typically based on first-arrival slope analysis ([4]). This interpretation technique is therefore less sensitive to errors in absolute traveltimes and zero-time corrections. Analogous to ZOPs, refracted air-wave arrivals can be misinterpreted as direct ground arrivals in case of nonzero surface offsets. The direct (first-arriving) wavefield can be significantly altered by interfering upgoing reflected wavefields, which leads to false first-break picks at and below the interface. Figure 1 a shows possible ray paths in the $\mathrm{ZOP}$ and the VRP geometries.

\section{B. Hydrogeologic setting and objectives}

Our investigations are based at the central Gnangara Mound, north of Perth, Western Australia. Aquifer recharge is controlled by winter rainfall and subsequent unsaturated flow through the unsaturated sandy soil profile of the Bassendean Formation ([14]). Our investigation focuses on the unsaturated zone and moisture distribution variations throughout the recharge period May to December 2011. The surface radar response of soil profiles of the Bassendean Sand Formation has been described by [11]. Due to cemented soil horizons within the podzolic soil profile (BH(s) horizons), the vertical water content distribution is highly variable $([2,11])$. Those layers are characterized by elevated water contents due to their water retention potential.

In this paper, we discuss various BHR acquisition geometries and compare results. Time-lapse acquisition is indicative of repeatability at profile depths where no changes are expected (i.e. the saturated zone).

\section{METHODS}

\section{A. Data acquisition}

We acquired crosswell and surface-to-hole BHR data before, during and after main recharge events. Here, we will compare datasets acquired at two test-sites. Date of acquisition and rainfall are given in Table I and Figure 2 C. Data was collected with the Mala ProEx 2 control unit and Mala's bistatic dipole slimhole antenna with a nominal centerfrequency of $100 \mathrm{MHz}$ (antenna length $\sim 1.5 \mathrm{~m}$ ). The feedpoints of the antennae have been chosen as midpoint in the

This work is funded by an ASEG research grant and a Water Corporation funded CHDG project, E. Strobach is sponsored by the Curtin International

Postgraduate Research Scholarship 
geometry assignment. We took the borehole collar and a fixed point at the cable head $0.85 \mathrm{~m}$ above the antennaes' feedpoints as reference for depth calculation. Prior to borehole measurements, antennae were launched in the air at constant distance to determine zero-time corrections. For this purpose, antennae were oriented in an upright position. Those measurements were repeated after the last borehole experiment had finished. ZOPs were acquired as explained in the introduction. For triggering we used the Mala borehole triggering wheel and the predefined Mala count-to-depth calibration setting which we found to be accurate and repeatable. Due to spikes in the data, we did not stack measurements during acquisition and decreased the spatial sampling interval to $1.5 \mathrm{~cm}$ instead (see processing section below). The temporal sampling interval was chosen to be 0.2 ns to obtain a well-sampled first-arriving wavelet.

\section{B. Data processing}

The borehole data showed some peculiarities. Spikes in the data at random positions prevented us from stacking data during acquisition. Even at high stack numbers (i.e. 256) S/N was greatly reduced. As described above, we increased the spatial sampling and applied several short windowed 2D median filters to remove the spikes. Data was then stacked to 8 $\mathrm{cm}$ trace increments. A second issue was that the data was clipped for the first arriving direct wavefield. In ZOP acquisition mode, clipping occurred at borehole separations of up to $11.5 \mathrm{~m}$ for the unsaturated zone ([12]), and at up to 4.75 $\mathrm{m}$ borehole separation in the saturated zone. Despite the high temporal sampling, satisfactory wavelet reconstruction could not be achieved with a third-order polynomial interpolation. Automatic first-break picking is expected to be influenced by clipped amplitudes and we decided to pick the first zerocrossing. For zero-crossing picking we re-sampled the data to 0.1 ns time increments. Despite the errors due to wavelet distortion, we believe that zero-cross picking provides the most accurate means of traveltime analysis in crosswell and surfaceto-hole radar if time-lapse measurements are to be compared.

Further processing included DC-shift correction (DC window before first-arrival) and static time shift (zero-time correction). We derived zero-time correction $t_{\text {corr }}$ by calculating the expected arrival of an air wave (velocity $v_{\text {air }}=0.3 \mathrm{~m} / \mathrm{ns}$ ) from antenna at known distance $\overline{d_{R x-T x}}$ and subtracted from the observed arrival time $t_{\text {obs. }}$ :

$$
t_{\text {corr }}=t_{\text {obs. }}-\frac{\overline{d_{R x-T x}}}{v_{\text {air }}}
$$

We chose the antenna distance in air to be consistent with the distance of the boreholes normalized by expected wavelength. High repeatability is essential for time-lapse experiments, especially for proximate boreholes (i.e. < $3 \mathrm{~m}$ separation) where small variations in traveltime lead to large variations in velocity, especially in the unsatured zone where traveltimes are small. We balanced ZOP traveltimes of subsequent months to bring velocities in the saturated zone to a common level. This can be done under the assumption that traveltimes in the saturated zone remain constant throughout the year and errors are contributed to inaccurate zero-time correction only. As a separate approach, we adjusted the ZOP traveltimes so that derived velocities are consistent with average VRP velocities in the unsaturated interval. With this method, however, ZOP and VRP velocities diverge in the saturated zone, which will be discussed in the following section.

We calculated water content from dielectric permittivities, which are in turn derived from velocity estimates under the assumption of lossless propagation. That is, the imaginary part of dielectric permittivity is negligible ([5]). Dielectric lab measurements performed in a coaxial transmission line showed that different sand and cemented horizon samples from the Bassendean Formation have a very low loss (i.e. negligible electrical conductivity and imaginary dielectric permittivity) and their water-content versus dielectric permittivity approximately follows the Topp - relationship.

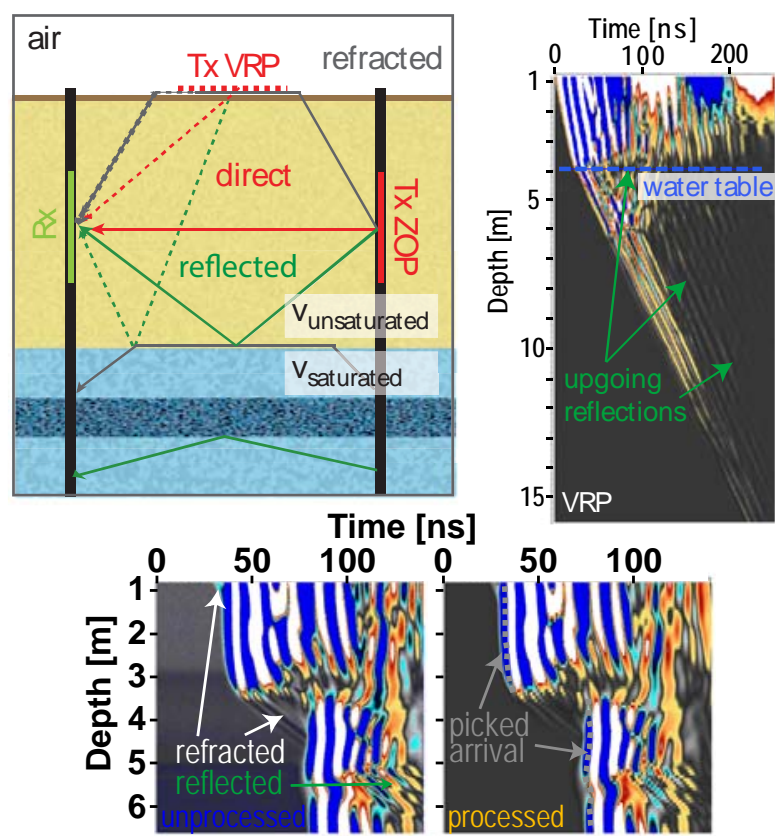

Figure 1. Upper: Schematic showing possible ray paths in VRP and ZOP geometry. Lower: Unprocessed (left) and processed ZOP example dataset from NG16. Note the blue spikes in the unprocessed data. The layer at $5.5 \mathrm{~m}$ depth apparently attenuates the signal in the ZOP which contradicts VRP data (upper right).

\section{Test Sites}

We analyse data from two test-sites, High-Hill Road West (HHW) (390360E/6508146N, MGA Zone 50, GDA 94), and boreholes NG16 (394849E/6519386N) at Airfield Rd. Borehole separations are $0.97 \mathrm{~m}$ and 4.75 at HHW and NG16, respectively. At HHW the borehole had a $0.6 \mathrm{~m}$ steel casing above ground. The soil profile at HHW is characterized by a deeply drained, layered sand with a "strong brown [...] weakly cohesive and consolidated" silty sand horizon at $1.4 \mathrm{~m}$ depth ([10]). The water table is at a depth of 11.5 - $11.8 \mathrm{~m}$ below natural surface (bNS). At NG16, the water table is shallow between $3.5-4 \mathrm{~m}$ bNS. The NG16 soil profile has been described as "white, fine to medium grained [...] quartz sand" between $0-5 \mathrm{~m}$ bNS and "brown [...] quartz sand [...] with weak to moderate ferruginous cementation (coffee rock)" between $5-7 \mathrm{~m}$ bNS ([8]). The cementation horizon is therefore below the water table. We found the cementation horizon to be at a depth of 5.4 - $6 \mathrm{~m}$ bNS and will discuss this interpretation in the next section. The borehole collar was even with the natural surface and no steel casing is present at NG16. 
TABLE I. TEST SiTE CHARACTERISTICS AND CUMULATIVE RAINFALL VALUES $\Sigma$ AND DIFFERENTIAL RAINFALL $\Delta$ BETWEEN SUBSEQUENT FIELD CAMPAIGNS

\begin{tabular}{|c|c|c|c|}
\hline \multicolumn{2}{|c|}{ Test-site } & High Hill Rd W & NG16 Airfield Rd \\
\hline \multicolumn{2}{|c|}{ Date (month \#) } & $5,6,8,9,10,12$ & $4,7,8,9$ \\
\hline \multicolumn{2}{|c|}{ Borehole separation [m] } & 0.98 & 4.85 \\
\hline \multicolumn{2}{|c|}{ Depth to watertable [m] } & $\begin{array}{l}11.6,11.7,11.7, \\
11.7,11.8,11.8\end{array}$ & $4,3.9,3.5,3.3$ \\
\hline \multicolumn{2}{|c|}{ Vegetation } & + & - \\
\hline \multicolumn{2}{|c|}{ Weather Station } & Gingin Aero & Gingin Aero \\
\hline 1st & $\Sigma[m m]$ & 19 & 7 \\
\hline 2nd & $\Sigma / \Delta[\mathrm{mm}]$ & $216 / 197$ & $246 / 239$ \\
\hline 3rd & $\Sigma / \Delta[\mathrm{mm}]$ & $392 / 176$ & $407 / 161$ \\
\hline 4th & $\Sigma / \Delta[\mathrm{mm}]$ & $514 / 122$ & $521 / 114$ \\
\hline 5th & $\Sigma / \Delta[\mathrm{mm}]$ & $637 / 123$ & - \\
\hline 6th & $\Sigma / \Delta[\mathrm{mm}]$ & $696 / 59$ & - \\
\hline
\end{tabular}

\section{RESULTS}

\section{A. Borehole Radar Techniques: ZOP and VRP compared}

HHW Time-lapse ZOP travel-time curves were highly repeatable. Although we found mismatch in absolute values between different months due to inconsistent zero-time corrections, the general shape of curves did not change in the saturated zone for both test-sites. At HHW, the shape of the traveltime curves did not change between 7.5 and $11 \mathrm{~m}$ throughout the unsaturated zone for all experiments. At NG16, the ZOP curve shapes were repeatable below the water table between $4 \mathrm{~m}$ and $6.5 \mathrm{~m}$ depth. The absolute misfit between subsequent month in this zone (i.e. saturated) was less severe. This can be explained by the increased borehole separation and increased absolute traveltimes, leading to a smaller relative error in zero-time correction (i.e. $t_{\text {corr }}<<$ traveltime). As a comparison, VRPs showed mixed results. We performed multioffset VRPs and found that $1 \mathrm{~m}$ and $2 \mathrm{~m}$ offsets provided most robust interval velocity estimation. In July, only a zero-offset VRP (feedpoint at borehole collar) was performed at HHW which suffered from poor coupling and subsequent polarity flips and high sensitivity to interference with upgoing reflected wavefields. The derived interval velocity profile shows inconsistent values at 6.5 and $9.6 \mathrm{~m}$ depth compared to the other months. The curves are generally repeatable, but seem to be noisier than the ZOPs. Also the location of maxima and minima is shifted to greater depth relative to the ZOP curves. The window for slope estimation was $1.2 \mathrm{~m}$. The general trend of major change in velocity, and the depth to which change took place in subsequent month, is similar to the ZOP interpretation. We found a large disparity between VRP- and ZOP-derived velocities throughout the unsaturated zone with ZOP velocities consistently smaller. When adjusting zero-time correction to match VRP and ZOP velocities in the saturated zone, the mismatch increases. The velocity estimation from local slopes of VRP first arrivals, although noisy, was quite robust for recovering absolute velocity and we trust those values. Dispersion in the material or changes in emitted waveform can lead to frequency shifts. Centroid frequency was observed to be lower in the saturated zone. By picking zerocrossings, however, we would obtain larger traveltimes for lower frequency wavelets (underestimate velocity) which contradicts our observation. We tried to pick first-arrivals instead of zero-crossings, but obtained very similar results. Because both, a shift in frequency and false zero-time correction cannot bring ZOP and VRP roughly in agreement, there might be an inherent problem with the ZOP interpretation for wells at sub-wavelength borehole separations. Alteration of the waveform due to different antenna coupling in different media (i.e. water filled borehole vs. dry hole and unsaturated medium) and near-field effects can explain the observed discrepancies. Variations in borehole separation are an alternative source of error and severe for small distances. Applied to our dataset, we could explain discrepancies between ZOP and VRP velocities by assuming larger distance where ZOP velocities are smaller than expected (i.e. between $6-11$ $\mathrm{m}$ ), and smaller distance where velocities are too large (i.e. saturated zone). For deviating boreholes, this explanation is therefore not appropriate. In theory, complex borehole geometry could explain VRP and ZOP discrepancies; however, we have no proof for this hypothesis.

NG16 In contrast to the comparatively wellbehaved VRP curves at HHW, the NG16 VRPs were less reliable (Figure $3 \mathrm{~b}$ ). To preserve detail in the unsaturated zone, we chose a window of $0.8 \mathrm{~m}$ to estimate local slope. Note that for July, a 4.85 m offset VRP was performed only. The August $1 \mathrm{~m}$ offset data shows great deviation from the other curves (i.e. between 4 and $6 \mathrm{~m}$ and 12, 13 and $14 \mathrm{~m}$ depth). In general, no clear trends are evident in the unsaturated zone when comparing same offsets from different months (not explicitly shown here). However, plotting all offset VRP interval velocity profiles (i.e. offsets between $1-8 \mathrm{~m}$ shown as one colour per month, Figure $3 \mathrm{~b}$ ) reveals a trend, similar to the ZOP observation. For qualitative interpretation, the VRPs might be of use. We do not have an explanation for the poor performance of the VRPs at NG16. When estimating velocity from absolute traveltimes rather than local slopes, the results look promising (not shown here). But this, again, requires very accurate zero-time correction which we found to be problematic, especially when dealing with close offsets in the near-field of the antenna. Another problem occurs due to electromagnetic energy travelling from antenna tip to tip. False velocity estimates due to dependency on raypath-angle from the horizontal are the result of that effect ([6]). At large offsets, refracted energy can become problematic.

Another feature we want to discuss at the NG16 site is the cemented soil horizon described in the previous section (lithology $\log 5-7 \mathrm{~m}$ ). The direct transmitted ZOP signal is attenuated from approximately $4.8-6.5 \mathrm{~m}$ depth, with smallest recorded amplitudes at $5.7 \mathrm{~m}$ bNS. Somewhat unexpected, coinciding with the amplitude minimum is a velocity maximum at $5.6 \mathrm{~m}$, possibly indicating lower porosity. Layer position and thickness can be estimated by assuming that the attenuation initiates when the lower antenna dipole (below the central feedpoint) enters the layer. Amplitudes increase when the upper antenna part exits the attenuating section. The onset of attenuation therefore starts half the antenna length deeper than the feedpoint position, and vice versa for the exit of the 
antenna. Where amplitudes are at their minimum, the antenna feedpoint and the layer midpoint are aligned. We estimate the onset of attenuation at $4.6 \mathrm{~m}$ in the data (feedpoint of antenna) which means at an actual depth of approximately $5.4 \mathrm{~m}$. Because the amplitudes do not make a full recovery before the borehole ends, we estimate layer thickness from the turn point where amplitudes increase at $5.7 \mathrm{~m}$ (feedpoint = layer midpoint). The layer is therefore approximately $0.6 \mathrm{~m}$ thick (2 $\mathrm{x} 0.3 \mathrm{~m}$ ). The damping of energy and decrease in traveltime coincides with a center-frequency shift of the recorded waveform from initially $100 \mathrm{MHz}$ to $70 \mathrm{MHz}$. The picked zero-crossing is biased towards greater traveltimes for lowfrequency waveforms and velocity is then underestimated. Picked first-arrivals show higher velocities in the attenuated zone. Higher velocities can be explained by increased cementation and therefore decreased porosity and hence reduced water content. However, we cannot explain the strong attenuation which is apparent in the ZOPs, but not in the VRP (Figure 1). The VRP shows a strong reflector at around $5.6 \mathrm{~m}$ bNS (estimated after wavefield separation in the f-k domain). A minor change in amplitudes of the first-arriving wavefield is evident below the reflection. The magnitude in amplitude reduction, however, can be solely contributed to reflection losses. In case of a conductive layer of $0.6 \mathrm{~m}$ thickness we would expected a larger decrease in amplitude in the VRP data. Further investigations need to be performed to explain the large response in the ZOP. Potential reasons are waveguide effects forcing EM waves to travel in the attenuating layer in case of the ZOP geometry. Local borehole effects coinciding with the depth interval of the cemented layer could also alter the local wavefield.

\section{B. Hydrogeologic observations and implications}

The following section will provide qualitative interpretation of time-lapse measurements and results should be understood as preliminary. No attempts are made to quantify unsaturated flow parameters. It has to be mentioned that winter rainfalls of previous years were greatly reduced compared to the long term average. As a result of that, soils are depleted in soil moisture, and vegetation deaths due to water stress have been recorded.

HHW Time-lapse radar velocity variations above $7.5 \mathrm{~m}$ (HHW), and above 3 - $3.5 \mathrm{~m}$ (NG16), are interpreted as being related to water content changes induced by water infiltration after rainfall. The drying in September is likely due to moisture uptake by vegetation. Figure 2 shows water content curves (Figure 2 A, B) alongside differential moisture curves (Figure $2 \mathrm{a}, \mathrm{b}$ ) that represent the difference of water content profiles between subsequent months. The curves depict the water front migrating through the soil profile. In June, the water front reached a depth of $2 \mathrm{~m}$ which coincides with a water retentive layer. For the following month, the changes in radar velocity indicate increasing moisture content between 1.5 - 4.5 m, 3 - 5.5 m, and $4-6.5 \mathrm{~m}$ in August, September and October, respectively. Approximately $30 \mathrm{~mm}$ of rainfall were recorded at a nearby weather station on the day of the October measurements. As a result, radar velocity decreased between 0 $-1 \mathrm{~m}$ bNS. Changes within $1-4 \mathrm{~m}$ depth are negligible and can be attributed to a slight depth-shift in the travel-time curves. The main water front continued to move downward. In December, no changes between depths of $4.5-6 \mathrm{~m}$ are evident. Velocities above $4.5 \mathrm{~m}$, however, increased indicating the onset of the drying period. Interestingly, parts of the profile at $1.4 \mathrm{~m}$ and $2.9 \mathrm{~m}$ bNS show no change from October to December, which coincides with unexpected drying in the previous month. This observation confirms a depth-shift in the October profile. In fact, the October data seems to be shifted downwards as peak values at $\sim 1.7 \mathrm{~m}$ and $3.2 \mathrm{~m}$ do not match other months. The differential moisture curves before calibration to unsaturated VRP velocities plotted in bright colours in Figure 2 a) show smaller, but similar (within $\sim 1.5$ vol\%) values compared to corrected curves plotted in dull coloration. Hence, changes can be quantified with acceptable error despite errors in absolute velocities .

NG16 The velocity variations at NG16 draw a similar picture. While high velocities in April represent the end of the dry season, the July data shows maximum annual moisture in the unsaturated zone. The test-site does not show water-retentive horizons in the unsaturated zone. At the large borehole distance, however, it is questionable whether variations would be expressed in first arrivals as significantly as in the closely spaced HHW crosswell data due to refracted energy ([12]). The ZOPs acquired in August indicate slight drying. A possible reason could be that measurements were performed after a short period of dry weather conditions (dark blue dotted line in Figure 2 C) opposed to measurements just after intensive rainfall in July. The same holds true for the September data. This observation could mean that the soil was above its field capacity in July and unsaturated flow took place, while August and September conditions were at equilibrium and soils at its field capacity. Note that the NG16 site was cleared, while vegetation was largely intact around HHW.

\section{CONCLUSIONS}

Vertical Radar Profiling and Zero (vertical-) Offset (crosswell) Profiling was performed at two test-sites during a winter recharge cycle in a sandy soil environment. Time-lapse measurements reveal moisture variations in the unsaturated zone due to water infiltration following natural precipitation. Our results point to strengths and weaknesses related to the different acquisition geometries VRP and ZOP. Our results can be summarized as follows:

1. ZOPs show good repeatability for the general shape of the velocity profiles, while VRP results were good for one test-site, but less robust at a second site.

2. ZOP absolute velocity determination is problematic when boreholes are close. Inaccuracy in borehole geometry (e.g. deviating holes) and zero-time correction can lead to large errors. Results can be verified and calibrated with supplementary VRP information.

3. For close boreholes, discrepancies between saturated and unsaturated zone can be large, possibly due to near-field effects (wavelength dependent) and antenna radiation characteristics. VRPs can be used for calibration, see 2 .

4. Differential water contents are not very sensitive to absolute travel-time errors, therefore very accurate 
zero-time correction or absolute velocity determination is not crucial in time-lapse investigations.

5. VRP slope analysis does not rely on absolute travel times and is therefore reliable in recovering absolute velocity. This information can be used for ZOP zerotime calibration (compare 2. and 3.).

6. VRP slope analysis can be unreliable for true zerooffset shots (Tx-feedpoint at borehole) due to bad coupling and strong interference effects from upgoing wavefields.

7. VRP slope analysis is more accurate for greater depth (i.e. large raypath angle from horizontal), offset VRPs can be unreliable for the shallow subsurface.

8. VRP first arrival determination can be influenced by interference with reflected (upgoing) wavefields. Wavefield separation (e.g. in fk-domain) can reduce the effect.

9. In the saturated zone at NG16, ZOPs show attenuation coinciding with a cemented layer. The VRPs confirm reflections from that horizon, but they do not show the same attenuation effect. Potential explanations are borehole or waveguide effects associated with the cemented layer.

For future investigations, more repeats during and after rainfall events will likely reveal moisture redistribution in more detail. Although observations at HHW suggest that no changes took place below $7 \mathrm{~m}$, shorter repeat intervals could confirm or discard this observation. At NG16, a plume-like movement of an infiltration front could not be observed. Intensive rainfall before July wet the soil to its field capacity. While saturation at field capacity persists, changes in moisture content can only be observed shortly after rainfall when water migrates to the water table. The soil then returns to its field capacity. In order to capture higher spatial detail and to overcome the potential nearfield problems for close wells, a higher frequency antenna configuration would be favorable.

Our study has demonstrated that borehole radar can be an integral tool to quantify water distribution and redistribution in a sandy soil profile during an annual recharge cycle.

\section{ACKNOWLEDGMENT}

We would like to thank Andrew Greendwood, Eva Caspari, Sinem Yavuz, Kostja Tertyshnikov, Majed Almalki and Franka Menzel for field assistance. Groundprobe Ltd. (Perth) kindly provided the borehole radar.

\section{REFERENCES}

[1] D. Alumbaugh, P.Y. Chang, L. Paprocki, J.R. Brainard, R.J. Glass, and C.A. Rautman. Estimating moisture contents in the vadose zone using cross-borehole ground penetrating radar: A study of accuracy and repeatability. Water Resour. Res, 38(12):1309, 2002.

[2] Muriel Bertuch and Ray Froend. Winter drawdown trial - whiteman park, soil characterisation report. Technical report, Edith-Cowan University, School of Natural Sciences, Perth, Western Australia, July 2006.

[3] A. Binley, P. Winship, R. Middleton, M. Pokar, and J. West. High-resolution characterization of vadose zone dynamics using cross-borehole radar. Water Resources Research, 37(11):2639-2652, 2001.

[4] William P. Clement and Michael D. Knoll. Traveltime inversion of vertical radar profiles. Geophysics, 71(3):K67-76, 2006.

[5] J. A. Huisman, S. S. Hubbard, J. D. Redman, and A. P. Annan. Measuring soil water content with ground penetrating radar: A review. Vadose Zone J., 2(4):476-491, 2003.

[6] James D. Irving, Michael D. Knoll, and Rosemary J. Knight. Improving crosshole radar velocity tomograms: a new approach to incorporating high-angle traveltime data. Geophysics, 72(4):J31-J41, July 2007.

[7] J.E. Peterson Jr. Pre-inversion corrections and analysis of radar tomographic data. Journal of Environmental and Engineering Geophysics, 6:1, 2001.

[8] J-P Pigois. North gnangara bore completion report. Department of Water, Western Australia, Hydrogeological record series, HR 277, 2010.

[9] Dale F. Rucker and Ty P. A. Ferre. Near-surface water content estimation with borehole ground penetrating radar using critically refracted waves. Vadose Zone Journal, 2(2):247-252, 2003.

[10] J. Searle and G. Bathols. Bore completion report for the shallow groundwater system investigation stage 2. Department of Water, Western Australia, Hydrogeological record series, HR 276, 2009.

[11] E. Strobach, B. D. Harris, J. C. Dupuis, A. W. Kepic, and M.W. Martin. Estimation of water content in partially saturated soil horizons with ground-penetrating radar. In 73rd EAGE Conference and Exhibition, 23-25 May 2011, Vienna, 2011.

[12] Elmar Strobach, Brett D. Harris, J. Christian Dupuis, Anton W. Kepic, and Michael W. Martin. Cross well radar and vertical radar profiling methods for time lapse monitoring of rainfall infiltration. 22nd ASEG Conference Extended Abstracts, 2012(1):1-4, February 2012.

[13] Jens Tronicke and Michael D. Knoll. Vertical radar profiling; influence of survey geometry on first-arrival traveltimes and amplitudes. Journal of Applied Geophysics, 57(3):179-191, April 2005.

[14] C. Xu, M. Canci, M. Martin, M. Donnelly, and R. Stokes. Perth regional aquifer modelling system (prams) model development: Application of the vertical flux model. Department of Water, Western Australia, Hydrogeological record series, HG 27, 2008. 


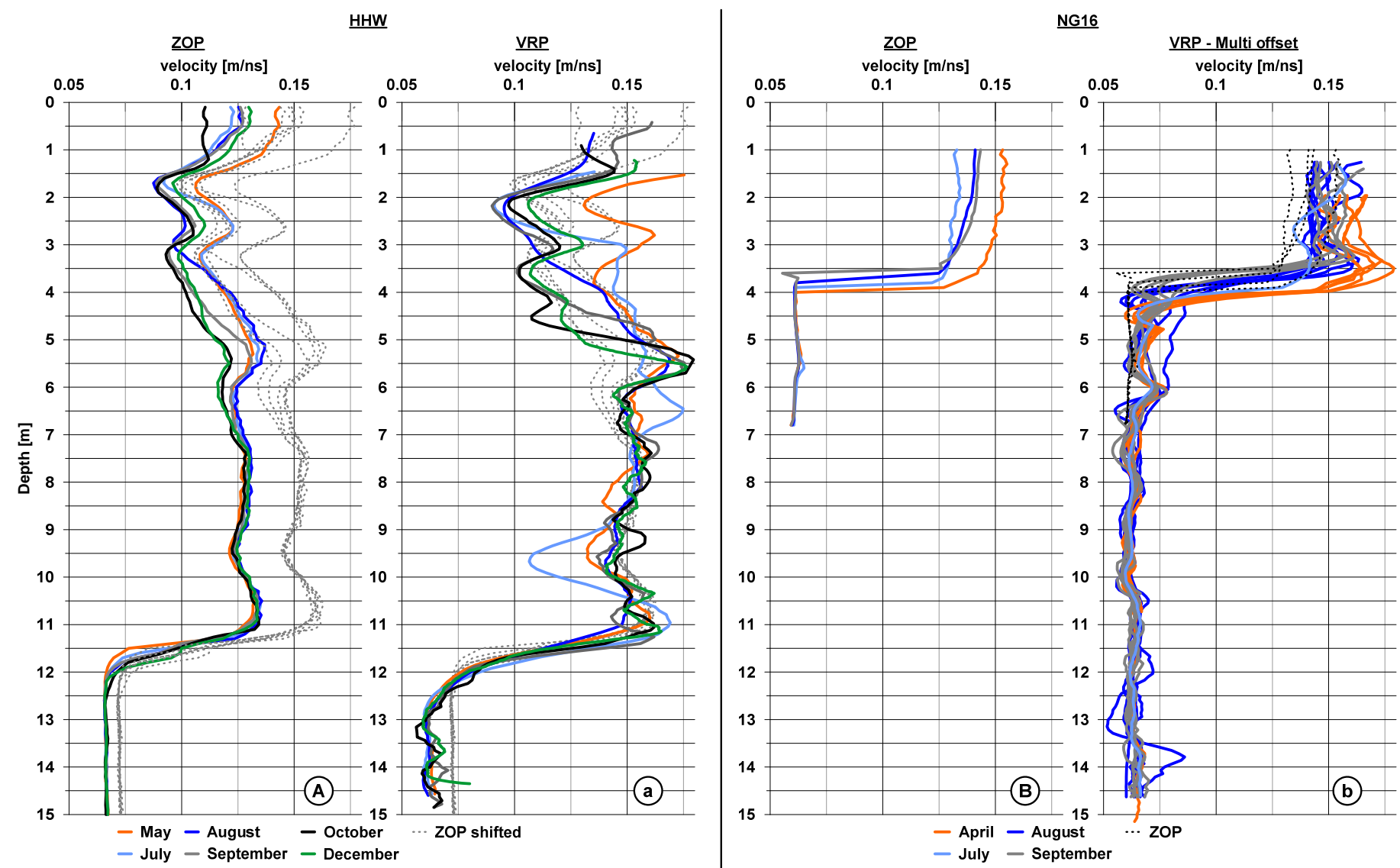

Figure 3. Time-lapse ZOP (left) and VRP (right) velocities compared for test-sites HHW (left) and NG16 (right). Borehole NG16c had a maximum depth of 7.5 $\mathrm{m}$, the VRP measurements were done in NG16b. For NG16 all offsets $(1 \mathrm{~m}-5 \mathrm{~m})$ VRPs are shown, individual offsets did not show clear trends. At HHW the July VRP is a zero-offset (Tx feedpoint directly over well), while other month are $1 \mathrm{~m}$ offsets. The gray dashed lines in HHW are the shifted ZOP curves to match VRP velocities in the unsaturated zone.

High Hill Rd West

NG16 Airfield Rd

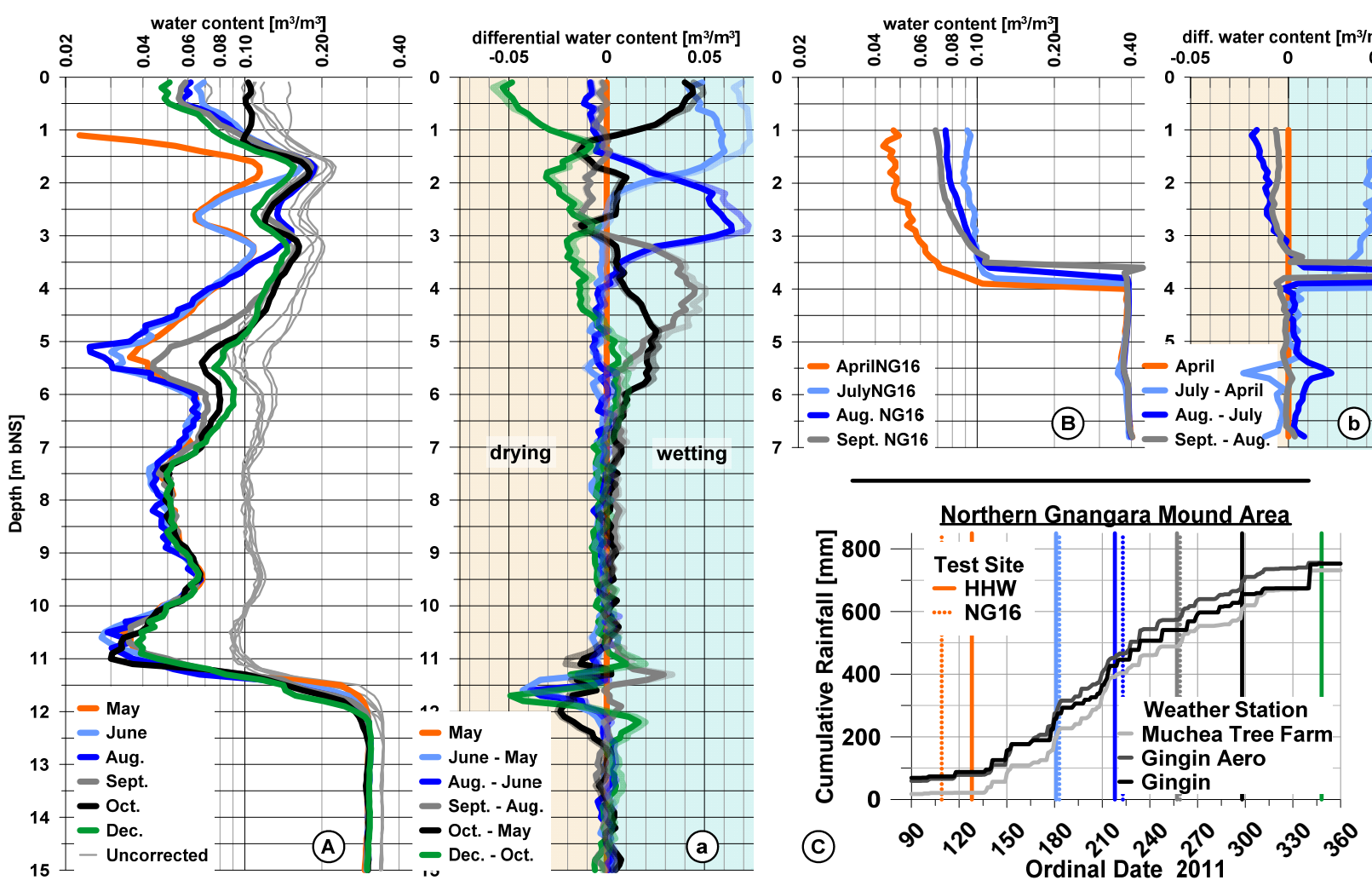

Figure 2. HHW and NG16 ZOP derived water content profiles and associated differential water content curves. Note the large difference in water content estimates between (VRP-) corrected ZOP water contents at HHW (gray lines in (A)), compared to the uncorrected water contents. Despite the large contrast between 6 and $11 \mathrm{~m}$ depth (section of most importance for unsaturated flow interpretation), the differential water content plots do not vary greatly (opaque (uncorrected) vs. transparent (VRP-corrected) in (a)). The diagram in the lower right corner displays the days of field campaigns and the cumulative rainfall recorded at nearby climate observatories (http://www.bom.gov.au/climate/data/). Note the near-surface water content increase between $0-1 \mathrm{~m}$ depth in HHW October data (black curve in (A),(a) and (C)) following approx. $30 \mathrm{~mm}$ of rainfall throughout all weather stations on the field day (ordinal date 298), while the depth interval between $1-4$ m shows a slight decrease for the retentive horizons. This observations indicates firstly that there is sensitivity for observing changes in the near-surface where antenna parts are above surface. Secondly. repeating time-lapse measurements more frequently after periods of intensive rainfall could capture higher detail of infiltration characteristics. 